\title{
Finitely Presented Modules over Right Non-Singular Rings.
}

\author{
UlRich Albrecht (*)
}

ABSTRACT - This paper characterizes the right non-singular rings $R$ for which $M / Z(M)$ is projective whenever $M$ is a cyclically (finitely) presented module. Several related results investigate right semi-hereditary rings.

\section{Introduction.}

The straightforward attempt to extend the notion of torsion-freeness from integral domains to non-commutative rings encounters immediate difficulties. To overcome these, one can concentrate on either the computational or the homological properties of torsion-free modules. Goodearl and others took the first approach when they introduced the notion of a nonsingular module [8]. A right $R$-module $M$ is non-singular if $Z(M)=0$ where $Z(M)=\{x \in M \mid x I=0$ for some essential right ideal $I$ of $R\}$ denotes the singular submodule of $M$. On the other hand, $M$ is singular if $Z(M)=M$. Moreover, a submodule $U$ of an $R$-module $M$ is $\mathcal{S}$-closed if $M / U$ is nonsingular. Finally, $R$ is a right non-singular ring if $R_{R}$ is non-singular.

The right non-singular rings are precisely the rings which have a regular, right self-injective maximal right ring of quotients, which will be denoted by $Q^{r}$ (see [8] and [11] for details). Following [11, Chapter XI], $Q^{r}$ is a perfect left localization of $R$ if $Q^{r}$ is flat as a right $R$-module and the multiplication map $Q^{r} \otimes_{R} Q^{r} \rightarrow Q^{r}$ is an isomorphism. In particular, $Q^{r}$ is a perfect left localization of $R$ if and only if every finitely generated nonsingular right $R$-module can be embedded into a projective module ([8] and [11]). We call a right non-singular ring with this property right strongly non-singular.

(*) Indirizzo dell'A.: Department of Mathematics, Auburn University, Auburn, AL 36849, U.S.A.

E-mail: albreuf@mail.auburn.edu 
Hattori took the second approach by defining $M$ to be torsion-free if $\operatorname{Tor}_{1}^{R}(M, R / R r)=0$ for all $r \in R$ [9]. The classes of torsion-free and nonsingular right $R$-modules coincide if and only if $R$ is a right Utumi p.p.-ring without an infinite set of orthogonal idempotents [3, Theorem 3.7]. Here, $R$ is a right p.p.-ring if all principal right ideals of $R$ are projective. Moreover, a right non-singular ring $R$ is a right Utumi-ring if every $\mathcal{S}$-closed right ideal of $R$ is a right annihilator.

Closely related to the notion of torsion-freeness are those of purity and relative divisibility. A sequence of right $R$-modules is pure-exact ( $R D$-exact) if every finitely presented (cyclically presented) module is projective with respect to it. Investigating $\mathrm{RD}$ - and pure-projective modules leads to the investigation of the condition that $M / Z(M)$ is projective. The dual question when $Z(M)$ is injective has been addressed in [8, Page 48, Example 24]. Section 2 discusses the question for which rings $M / Z(M)$ is projective for all RD-projective modules $M$. Theorem 2.1 shows that, provided $R$ has no infinite set of orthogonal idempotents, these are precisely the right Utumi p.p.rings discussed in [3]. The structure of pure-projective right $R$-modules was described in [4] in case that $R$ is a right strongly non-singular, right semihereditary ring $R$ without an infinite set of orthogonal idempotents. Theorem 2.3 shows that these conditions on $R$ are not only sufficient, but also necessary for the structure-theorem (part b) of Theorem 2.3 to hold.

Prüfer domains can be characterized as the domains with the property that, whenever a torsion-free module $M$ contains a projective submodule $U$ with $M / U$ finitely generated, then $M$ is projective and $M / U$ is finitely presented [7, Chapter VI]. We show that the right non-singular rings having the corresponding property for non-singular modules are precisely the right strongly non-singular, right semi-hereditary rings of finite right Goldie dimension (Theorem 3.1).

Section 4 investigates pure-projective modules over right hereditary rings. As part of our discussion, we obtain a characterization of the right Noetherian right hereditary rings with the restricted right minimum condition which are right strongly non-singular. The last results of this paper demonstrate that right invertible submodules of $Q_{R}^{r}$ which were introduced in [11, Chapters II.4 and IX.5] may fail to share many of the important properties of invertible modules over integral domains. For instance, the lattice of finitely generated right ideals over a Prüfer domain is distributive, i.e. $I \cap(J+K)=(I \cap J)+(I \cap K)$ for all finitely generated ideals $I, J$, and $K$ of $R$ [7, Theorem III.1.1]. Example 4.7 shows that there exists a right strongly non-singular, hereditary, right and left Noetherian ring whose finitely generated right ideals do not have this property. 


\section{RD-Projective Modules.}

Let $U$ be a submodule of a non-singular module $M$. The $\mathcal{S}$-closure of $U$ in $M$ is the submodule $V$ of $M$ which contains $U$ such that $V / U=Z(M / U)$.

THEOREM 2.1. The following are equivalent for a right non-singular ring $R$ without an infinite set of orthogonal idempotents:

a) $R$ is a right Utumi p.p.-ring.

b) $M / Z(M)$ is projective for every RD-projective module $M$.

Proof. $\quad a) \Rightarrow b$ ): Since every RD-projective module is a direct summand of a direct sum of cyclically presented modules, it suffices to verify b) in case that $M \cong R / a R$ for some $a \in R$. Let $J$ be the right ideal of $R$ which contains $a R$ such that $J / a R=Z(R / a R)$. Then, $R / J \cong M / Z(M)$ is a nonsingular cyclic module which is projective by [3, Corollary 3.4].

$b) \Rightarrow a)$ : Let $I$ be the $\mathcal{S}$-closure of $r R$ for some $r \in R$. Since $R / r R$ is RDprojective, and $R / I \cong(R / r R) / Z(R / r R)$, we obtain that $R / I$ is projective. Thus, $I$ is generated by an idempotent.

By [3, Lemma 3.5], it suffices to show that every $\mathcal{S}$-closed right ideal $J$ of $R$ is generated by an idempotent. For this, select $0 \neq r_{0} \in J$. Since $J$ is $\mathcal{S}$-closed in $R$, it contains the $\mathcal{S}$-closure $I_{0}$ of $r_{0} R$. By what has been shown so far, $I_{0}=e_{0} R$ for some idempotent $e_{0}$ of $R$. Hence, $J=e_{0} R \oplus\left[J \cap\left(1-e_{0}\right) R\right]$. If $J \cap\left(1-e_{0}\right) R \neq 0$, select a non-zero $r_{1}=\left(1-e_{0}\right) r_{1} \in J$; and observe that $J \cap\left(1-e_{0}\right) R$ is $\mathcal{S}$-closed in $R$. Hence, it contains the $\mathcal{S}$-closure $I_{1}$ of $r_{1} R$ in $R$. By the previous paragraph, $I_{1}=f R$ for some idempotent $f$ of $R$. Write $f=\left(1-e_{0}\right) s$ for some $s \in R$, and set $e_{1}=f\left(1-e_{0}\right)$. Since $e_{0} f=0$, we have $e_{1} e_{0}=e_{0} e_{1}=0$ and $e_{1}^{2}=f^{2}-f^{2} e_{0}-f e_{0} f+\left(f e_{0}\right)^{2}=f-f e_{0}=e_{1}$. Thus, $e_{0}$ and $e_{1}$ are non-zero orthogonal idempotents with $e_{1} R \subseteq f R$. On the other hand, $f=f\left(1-e_{0}\right) s=e_{1} s$ yields $f R=e_{1} R$. Consequently, $R=e_{0} R \oplus e_{1} R \oplus\left[J \cap\left(1-e_{0}-e_{1}\right) R\right]$. Continuing inductively, we can construct non-zero orthogonal idempotents $e_{0}, \ldots, e_{n+1} \in J$ as long as $J \cap\left(1-e_{0}-\ldots-e_{n}\right) R \neq 0$. Since $R$ does not contain an infinite family of orthogonal idempotent, this process has to stop, say $J \cap\left(1-e_{0}-\ldots-e_{n}\right) R=0$. Then, $e_{0}+\ldots+e_{m}$ is an idempotent with $J=\left(e_{0}+\ldots+e_{m}\right) R$.

We now investigate which conditions $R$ has to satisfy to ensure the validity of the structure theorem for pure-projectives in [4]. We want 
to remind the reader that a right $R$-module is essentially finitely generated if contains an essential, finitely generated submodule.

LEMma 2.2. The following are equivalent for a right non-singular $\operatorname{ring} R$ : mension.

a) $R$ is right semi-hereditary and has finite right Goldie-di-

b) A finitely generated right $R$-module $M$ is finitely presented if and only if p.d.M $\leq 1$.

Proof. $\quad a) \Rightarrow b$ ): Since $R$ is right semi-hereditary, every finitely presented module has projective dimension at most 1 . Conversely, whenever $M \cong R^{n} / U$ for some projective module $U$, then $U$ is essentially finitely generated since $R$ has finite right Goldie dimension. By Sandomierski's Theorem [5, Proposition 8.24], essentially finitely generated projective modules are finitely generated.

$b) \Rightarrow a$ ): Clearly, $R$ has to be right semi-hereditary. If $R$ has infinite right Goldie-dimension, then it contains a family $\left\{I_{n}\right\}_{n<\omega}$ of non-zero, finitely generated right ideals whose sum is direct. Since $R$ is right semihereditary, each $I_{n}$ is projective, and the same holds for $\oplus_{n<\omega} I_{n}$. By b), $R / \oplus_{n<\omega} I_{n}$ is finitely presented, a contradiction.

THEOREM 2.3. The following conditions are equivalent for a right nonsingular ring $R$ without an infinite set of orthogonal idempotents:

a) $R$ is a (right Utumi), right semi-hereditary ring such that $Q^{r}$ is a perfect left localization of $R$.

b) A right $R$-module $M$ is pure-projective if and only if

i) $Z(M)$ is a direct summand of a direct sum of finitely generated modules of projective dimension at most 1 .

ii) $M / Z(M)$ is projective.

Proof. A right strongly non-sigular, right semi-hereditary ring without an infinite set of orthogonal idempotents is a right Utumi ring [3, Theorems 3.7 and 4.2$]$.

$a) \Rightarrow b$ ): By [3], $R$ has finite right Goldie-dimension. Because of Lemma $2.2, Z(M)$ is pure-projective if and only if condition i) in b) holds. It remains to show that $M / Z(M)$ is projective whenever $M$ is finitely presented. However, this follows from [11] since $M / Z(M)$ is a finitely generated nonsingular module, and $Q^{r}$ is a perfect left localization of $R$. 
$b) \Rightarrow a$ ): To see that $R$ is right semi-hereditary, consider a finitely generated right ideal $I$ if $R$. The $\mathcal{S}$-closure $J$ of $I$ satisfies $J / I=Z(R / I)$. Hence, $R / J$ is projective by $b$ ), and $J / I$ has projective dimension at most 1 . Since $J$ is projective, this is only possible if $I$ is projective.

To show that $R$ has finite right Goldie-dimension, consider a right ideal of $R$ of the form $a_{0} R \oplus \ldots \oplus a_{n} R \oplus \ldots$ where each $a_{n} \neq 0$. For $m<\omega$, let $I_{m}$ be the $\mathcal{S}$-closure of $a_{0} R \oplus \ldots a_{m} R$. Since $R / I_{m}$ is projective by b), $I_{m}$ is generated by an idempotent $e_{m}$ of $R$. Write $I_{m+1}=I_{m} \oplus\left[I_{m+1} \cap\left(1-e_{m}\right) R\right]$. Observe that $\left[I_{m+1} \cap\left(1-e_{m}\right) R\right]$ is generated by an idempotent $f$ of $R$ as a direct summand of $R$. Setting $d_{m}=f\left(1-e_{m}\right)$ yields an idempotent $d_{m}$ of $R$ such that $e_{m} d_{m}=d_{m} e_{m}=0$, and $I_{m+1}=I_{m} \oplus d_{m} R$ as in the proof of Theorem 2.1. Inductively, one obtains an infinite family of orthogonal idempotents $\left\{d_{m} \mid m<\omega\right\}$ of $R$, which is not possible. Thus, $R$ has finite right Goldiedimension; and every $\mathcal{S}$-closed right ideal $J$ of $R$ is the $\mathcal{S}$-closure of a finitely generated right ideal. By b), $R / J$ is projective; and $R$ is a right Utumi-ring since $J=e R$ for some idempotent $e$ of $R$.

To establish that $Q^{r}$ is a perfect left localization of $R$, it suffices to show that every finitely generated non-singular right $R$-module $M$ is projective. Write $M \cong R^{n} / U$ and observe that $U$ is essentially finitely generated. Select a finitely generated essential submodule $V$ of $U$. Then, $U / V=Z\left(R^{n} / U\right)$, and $M$ is projective by b).

In the following, the injective hull of a module $M$ is denoted by $E(M)$.

COROLLARY 2.4. Let $R$ be a right semi-hereditary ring of finite right Goldie-dimension such that $Q^{r}$ is a perfect left localization of $R$. A right $R$ module $M$ is pure-projective if and only if $M / Z(M)$ is projective and $Z(M)$ is isomorphic to a direct summand of a direct sum of finitely generated submodules of $\left(Q^{r} / R\right)^{n}$.

Proof. We first show that a finitely generated singular module $M$ has projective dimension at most 1 if and only if it can be embedded into a finite direct sum of copies of $Q^{r} / R$. If $p . d . M \leq 1$, then there exist a finitely generated free module $F$ and an essential projective submodule $P$ of $F$ with $M \cong F / P$. Since $R$ has finite right Goldie-dimension, $P$ is essentially finitely generated, and hence itself finitely generated by Sandomierski's Theorem [5, Proposition 8.24]. We can find a finitely generated projective module $U$ such that $P \oplus U$ is a finitely generated free module, say $P \oplus U \cong R^{n}$. Since $M$ is singular, $P \oplus U$ is an essential submodule of $F \oplus U$. Therefore, $F \oplus U \subseteq E(P \oplus U)=\left(Q^{r}\right)^{n}$, and $M \subseteq\left(Q^{r} / R\right)^{n}$. 
On the other hand, if $M$ is a finitely generated submodule of $\left(Q^{r} / R\right)^{n}$, then there is a finitely generated submodule $U$ of $\left(Q^{r}\right)^{n}$ containing $R^{n}$ such that $M=U / R^{n}$. Since $R$ is right semi-hereditary, and since $Q^{r}$ is a perfect left localization of $R, U$ is projective and p.d.M $\leq$. The corollary now follows directly from Theorem 2.3.

\section{Essential Extensions of Projective Modules.}

In the commutative setting, Prüfer domains are characterized by conditions b) and c.ii) [7].

THEOREM 3.1. The following are equivalent for a right non-singular $\operatorname{ring} R$ :

a) $R$ is a right semi-hereditary ring of finite right Goldie-dimension for which $Q^{r}$ is a perfect left localization of $R$.

b) Whenever a non-singular module $M$ contains a projective submodule $U$ such that $M / U$ is finitely generated, then $M$ is projective and $M / U$ is finitely presented.

c) i) $R$ is a right p.p.-ring.

ii) If a finitely generated non-singular right $R$-module $M$ contains an essential projective submodule $U$, then $M$ is projective, and $M / U$ is finitely presented.

Proof. $\quad a) \Rightarrow b$ ): Let $W$ be the $\mathcal{S}$-closure of $U$ in $M$. Since $M / W$ is finitely generated as an image of $M / U$ and non-singular, it is projective by a). Hence, $M=W \oplus P$ for some finitely generated projective module $P$. We may thus assume that $M / U$ is singular.

Since $R$ is right semi-hereditary, $U=\oplus_{I} U_{i}$ where each $U_{i}$ is finitely generated [1]. Because $M / U$ is singular and $M$ is non-singular, $U$ is essential in $M$. Thus, $E(M)=E(U)=\oplus_{I} E\left(U_{i}\right)$ in view of the fact that direct sums of non-singular injectives are injective if $R$ has finite right Goldie-dimension [11, Proposition XIII.3.3]. Choose a finitely generated submodule $V$ of $M$ such that $M=U+V$. There is a finite subset $J$ of $I$ such that $V \subseteq \oplus_{J} E\left(U_{i}\right)$. Then, $W_{1}=V+\oplus_{J} U_{i}$ is a finitely generated submodule of $\oplus_{J} E\left(U_{i}\right)$ such that $V \cap\left(\oplus_{I \backslash J} U_{i}\right)=0$. Consequently, $M=W_{1} \oplus \oplus_{I \backslash J} U_{i}$. But $W_{1}$ is projective by a) showing that $M$ is projective and that $M / U=W_{1} / U$ is finitely presented.

$b) \Rightarrow c)$ : Observe that every finitely generated non-singular module is projective by choosing $U=0$ in b). 
$c) \Rightarrow a$ ): Assume that $R$ contains a right ideal $U$ of the form $U=\oplus_{n<\omega} a_{n} R$ where each $a_{n} \neq 0$. By part i) of c), $U$ is projective. Choose a right ideal $V$ of $R$ which is maximal with respect to the property $U \cap V=0$. Since $R$ is right non-singular, $V$ is an $\mathcal{S}$-closed submodule of $R$ and $[R / V] /[U \oplus V / V] \cong R /(U \oplus V)$ is singular. Therefore, the projective module $U \oplus V / V$ is essential in the non-singular module $R / V$. By c), $R / V$ is projective; and $R /(U \oplus V)$ is finitely presented. Hence, $U$ is finitely generated which is not possible.

To see that $R$ is a right strongly non-singular, right semi-hereditary ring, it suffices to show that a finitely generated non-singular right $R$ module $M$ is projective. By [11, Proposition XII.7.2], $M \subseteq\left(Q^{r}\right)^{n}$ for some $n<\omega$. Since $R$ has finite right Goldie dimension and $R^{n}$ is essential in $\left(Q^{r}\right)^{n}, M$ has finite Goldie-dimension. Therefore, $M$ contains uniform submodules $U_{1}, \ldots, U_{m}$ such that $U_{1} \oplus \ldots \oplus U_{m}$ is essential in $M$. Furthermore, we may assume that each $U_{i}$ is cyclic, say $U_{i}=b_{i} R$. Since $M$ is non-singular, $a n n_{r}\left(b_{i}\right)=\left\{r \in R \mid b_{i} r=0\right\}$ is not essential in $R$. Select $c_{i} \in R$ with $c_{i} R \cap a n n_{r}\left(b_{i}\right)=0$. Then, $U_{i}$ contains a submodule $V_{i} \cong c_{i} R$. Since $R$ is a right p.p.-ring, $V_{i}$ is projective. Hence, $M$ contains the essential projective submodule $V_{1} \oplus \ldots \oplus V_{m}$. By c), $M$ is projective.

A submodule $U$ of a module $M$ is tight if both $U$ and $M / U$ have projective dimension at most 1 . A module is coherent if all its finitely generated submodules are finitely presented.

COROLLARY 3.2. Let $R$ be a right semi-hereditary ring of finite right Goldie-dimension such that $Q^{r}$ is a perfect localization of $R$.

a) $A$ right $R$-module $M$ of projective dimension at most 1 is coherent. Moreover, all its finitely generated submodules are tight.

b) If $M$ is singular and a direct sum of countably generated modules, then p.d.M $\leq 1$ if and only if $M \subseteq\left(Q^{r} / R\right)^{(I)}$ for some index-set $I$.

Proof. a) Write $M \cong F / P$ where $F$ and its submodule $P$ are projective. If $U$ is a finitely generated submodule of $M$, then there is a submodule $W$ of $F$ containing $P$ with $W / P \cong U$. By Theorem 3.1, $W$ is projective and $W / P$ is finitely presented. Clearly, $U$ and $M / U$ have projective dimension at most 1 .

b) Without loss of generality, we may assume that $M$ is countably generated. If $p . d . M \leq 1$, then $M=F / P$ where $F$ is projective and $P \cong R^{(I)}$ for some index-set $I$. Since $P$ is essential in $F$, we have $F \subseteq E(P) \cong\left(Q^{r}\right)^{(I)}$ 
by [11, Proposition XIII.3.3] because $R$ has finite right Goldie-dimension. Hence, $M \subseteq\left(Q^{r} / R\right)^{(I)}$.

Conversely, suppose that $M \subseteq\left(Q^{r} / R\right)^{(\omega)}$, and select a submodule $U$ of $\left(Q^{r}\right)^{(\omega)}$ containing $R^{(\omega)}$ such that $M=U / R^{(\omega)}$. Choose $\left\{u_{n} \mid n<\omega\right\} \subseteq U$ such that $U=\Sigma_{n<\omega} u_{n} R+R^{(\omega)}$ and $u_{0}=0$. Set $V_{\ell}=R^{(\omega)}+\Sigma_{n=1}^{\ell} u_{n} R$. By Theorem 3.1, each $V_{\ell}$ is projective. Let $W_{\ell}=V_{\ell} / R^{(\omega)} \subseteq M$. Then, $W_{0}=0$ and $M=\cup_{n=1}^{\omega} W_{n}$. Observe that $W_{\ell+1} / W_{\ell} \cong V_{\ell+1} / V_{\ell}$ has projective dimension at most 1. By Auslander's Theorem, p.d.M $\leq 1$.

\section{Hereditary Rings.}

The first result describes the right strongly non-singular, right Noetherian, right hereditary rings.

Proposition 4.1. The following conditions are equivalent for a right non-singular ring $R$ of finite right Goldie dimension:

a) $R$ is a right strongly non-singular, right hereditary ring without an infinite set of orthogonal idempotents.

b) $R$ is a right strongly non-singular, right Noetherian and right hereditary.

c) i) $R$ has finite right Goldie dimension.

ii) $M$ is pure projective if and only if $M / Z(M)$ is projective, and $Z(M)$ is a direct summand of a direct sum of finitely generated modules.

Proof. $\quad a) \Rightarrow b$ ): By [3, Theorems 3.7 and 4.2], $R$ has finite right Goldie dimension. However, essentially finitely generated projective modules are finitely generated [5, Proposition 8.24]. $b) \Rightarrow c$ ) is obvious in view of Theorem 2.3.

$c) \Rightarrow a$ ): Let $I$ be a right ideal of $R$, and $J$ its $\mathcal{S}$-closure in $R$. Since $R$ has finite right Goldie dimension, $I$ contains a finitely generated right ideal $K$ as an essential submodule. Thus, $J$ is the $\mathcal{S}$-closure of $K$, and $J / K$ is the singular submodule of the finitely presented module $R / K$. By c), $R / J$ is projective, and $R=J \oplus J_{1}$. Then, $R / I \cong J / I \oplus J_{1}$. In particular, $J / I$ is a finitely generated singular module, which is pure-projective by c). Hence, $J / I$ is a direct summand of a direct sum of finitely presented modules. Clearly, this sum can be chosen to be finite. Therefore, $J / I$ is finitely presented, and $I$ is finitely generated since $J$ is a direct summand of $R$. Once we have shown that every finitely generated non-singular right $R$ - 
module $M$ is projective, we will have established that $R$ is a right hereditary ring with the property that $Q^{r}$ is a perfect left localization of $R$.

There exists a finitely generated free module $F$ and a submodule $U$ of $F$ such that $M \cong F / U$. Since $R$ has finite right Goldie-dimension, $U$ contains a finitely generated essential submodule $V$. Because, $F / U$ is non-singular, $U / V$ is the singular submodule of the finitely presented module $F / V$. By c), $F / U \cong(F / V) /(U / V)$ is projective.

COROLLARY 4.2. The following are equivalent for a right non-singular ring $R$ with finite right Goldie-dimension:

a) $R$ is a right Noetherian, right hereditary ring which satisfies the restricted right minimum condition such that $Q^{r}$ is a perfect left localization of $R$.

b) $M$ is pure projective if and only if $M / Z(M)$ is projective and $Z(M)$ is a direct summand of a direct sum of finitely generated Artinian modules.

Proof. $\quad a) \Rightarrow b$ ): Since $R$ has the restricted minimum condition, every finitely generated singular right module is Artinian.

$b) \Rightarrow a)$ : Let $I$ be an essential right ideal of $R$. Since $R$ has finite right Goldie-dimension, $I$ contains a finitely generated essential right ideal $J$. By c), the finitely presented module $R / J$ is a direct summand of a (finite) direct sum of finitely generated Artinian modules. But this is only possible if $R / J$ is Artinian. But then, $R / I$ is Artinian too. Arguing as in the proof of Proposition 4.1, we obtain that $R$ is a right strongly non-singular, right hereditary.

By [8, Proposition 5.27], a right hereditary, right Noetherian ring which is the product of prime rings and rings Morita equivalent to lower triangular matrix rings over a division algebra is right strongly non-singular and has the restricted right minimum condition, i.e. $R / I$ is Artinian for every essential right ideal $I$ of $R$.

Let $U$ be a subset of $Q^{r}$, and set $(R: U)_{r}=\left\{q \in Q^{r} \mid U q \subseteq R\right\}$ and $(R: U)_{\ell}=\left\{q \in Q^{r} \mid q U \subseteq R\right\}$.

THEOREM 4.3. The following are equivalent for a ring $R$ :

a) $R$ is a right Noetherian right hereditary ring satisfying the restricted right minimum condition such that $Q^{r}$ is a perfect left localization of $R$. 
b) $R$ is a left Noetherian left hereditary ring satisfying the restricted left minimum condition such that $Q^{\ell}$ is a perfect right localization of $R$.

c) $R$ is a right and left Noetherian, hereditary, right and left Utumi-ring.

d) $R=R_{1} \times \ldots \times R_{n}$ where each $R_{i}$ is either a prime right and left Noetherian hereditary ring or Morita equivalent to a lower triangular matrix ring over a division algebra.

Proof. $\quad a) \Rightarrow c$ ): By [3, Theorem 4.2], $Q^{r}=Q^{\ell}$ is a semi-simple Artinian ring, and $R$ is right and left Utumi. Furthermore, $R$ is left semi-hereditary by [3, Theorem 5.2]. It remains to show that $R$ is left Noetherian.

Suppose that $R$ contains a left ideal $I$ which is not finitely generated. Without loss of generality, we may assume that $I$ is essential in $R$. Since $Q^{r}=Q^{\ell}$ is semi-simple Artinian, $R$ has finite left Goldiedimension [11, Theorem XII.2.5], and $I$ contains a finitely generated essential left ideal $J_{0}$. Since $I$ is not finitely generated, we can find an ascending chain $J_{0} \subseteq \ldots \subseteq J_{n} \subseteq \ldots$ of finitely generated essential left ideals inside $I$ with $J_{n} \neq J_{n+1}$ for all $n$.

Since $Q^{r}$ is an injective left $R$-module being the maximal left ring of quotients of $R$, every map $\phi: J_{i} \rightarrow Q^{r}$ is right multiplication by some $q \in Q^{r}$, which is uniquely determined by $\phi$ since $J_{i}$ is essential. Therefore, we can identify $\operatorname{Hom}_{R}\left(J_{i}, R\right)$ and $J_{i}^{*}=\left(R: J_{i}\right)_{r}$. Moreover, $J_{i}^{*}$ is a finitely generated projective right $R$-module because $J_{i}$ is projective since $R$ is left semi-hereditary. Furthermore, $J_{i}^{* *}=\left(R: J_{i}^{*}\right)_{\ell}$ satisfies $J_{i}^{* *}=J_{i}$. To see this, observe that $J_{i} \subseteq J_{i}^{* *}$ by definition. Conversely, $J_{i}$ has a finite projective basis since it is finitely generated and projective. There are $a_{1}, \ldots, a_{k} \in J_{i}$ and $q_{1}, \ldots, q_{k} \in J_{i}^{*}$ such that $y=y q_{1} a_{1}+\ldots+y q_{k} a_{k}$ for all $y \in J_{i}$. Since $Q^{r}$ also is the maximal left ring of quotients of $R$, it is non-singular as a left $R$-module. Because $J_{i}$ is an essential left ideal, $1=q_{1} a_{1}+\ldots+q_{k} a_{k}$. If $z \in J_{i}^{* *}$, then $z q_{i} \in R$, and $z=\left(z q_{1}\right) a_{1}+\ldots\left(z q_{k}\right) a_{k} \in J_{i}$.

We obtain a descending chain $J_{0}^{*} \supseteq \ldots \supseteq J_{n}^{*} \supseteq \ldots \supseteq R^{*}=R$ of finitely generated submodules of $Q_{R}^{r}$. Since $Q^{r} / R$ is singular, $J_{0}^{*} / R$ is a finitely generated singular right $R$-module. By the restricted right minimum condition for $R, J_{0}^{*} / R$ is Artinian. Consequently, there is $m$ with $J_{m}^{*}=J_{m+1}^{*}$, from which one obtains $J_{m}=J_{m+1}$.

$c) \Rightarrow d$ ): By [3, Theorem 5.2], the classes of torsion-free, non-singular and flat right $R$-modules coincide. Because of [3, Theorem 5.5], $R$ has the desired form. 
$d) \Rightarrow a)$ : By [3, Theorem 5.5], $R$ is a right and left Noetherian hereditary ring for which the classes of torsion-free, flat and non-singular modules coincide. Thus, $Q^{r}$ is a perfect left localization of $R$ by [3, Theorem 5.2]. Finally, $R$ satisfies the restricted right minimum condition by [5].

Since condition c) is right-left symmetric, the equivalence of a) and b) follows immediately.

A submodule $U$ of the right $R$-module $Q^{r}$ is right invertible if there exist $u_{1}, \ldots, u_{n} \in U$ and $q_{1}, \ldots, q_{n} \in(R: U)_{\ell}$ with $u_{1} q_{1}+\ldots u_{n} q_{n}=1$ [11, Chapters ii.4 and IX.5]. Over an integral domain $R$, a right invertible module $U$ has the additional property that $U(R: U)_{\ell}=R$ which may fail in the non-commutative setting:

ExAMPLE 4.4. There exists a right and left Artinian, hereditary ring $R$ such that $Q^{r}$ is a perfect right and left localization of $R$, which contains an essential right ideal $I$ with $(R: I)_{\ell}=Q^{r}$ and $(R: I)_{\ell} I=I$. Moreover, there exist $r_{1}, r_{2}, s_{1}, s_{2} \in I$ and $q_{1}, q_{2}, p_{1}, p_{2} \in(R: I)_{\ell}$ satisfying $1=r_{1} p_{1}+r_{2} p_{2}=$ $=s_{1} q_{1}+s_{2} q_{2}$ such that $r_{1} p_{1}, r_{2} p_{2} \in R$, but $s_{1} q_{1}, s_{2} q_{2} \notin R$. Thus, $I(R: I)_{\ell} \nsubseteq R$.

Proof. Let $F$ be a field of characteristic different from 2 , and $R$ be the lower triangular matrix ring over $F$. Clearly, $R$ is a right and left Artinian ring. According to [8, Theorem 4.7], $R$ is a right hereditary ring, which is also left hereditary by [5, Corollary 8.18]. Finally, by [8, Proposition 2.28 and Theorem 2.30], the maximal right and left ring of quotients of $R$ is $Q=\operatorname{Mat}_{2}(F)$. Inside $R$, we consider the idempotents $e_{1}=\left(\begin{array}{ll}1 & 0 \\ 0 & 0\end{array}\right)$ and $e_{2}=\left(\begin{array}{ll}0 & 0 \\ 0 & 1\end{array}\right)$. Let $I=\left(\begin{array}{ll}F & 0 \\ F & 0\end{array}\right)$, a two-sided ideal of $R$ which is essential as a right ideal because $\left(\begin{array}{ll}a & 0 \\ b & c\end{array}\right)\left(\begin{array}{ll}0 & 0 \\ 1 & 0\end{array}\right)=\left(\begin{array}{ll}0 & 0 \\ c & 0\end{array}\right)$ for all $a, b, c \in F$. Since $I=Q^{r} e_{1}$, we have $(R: I)_{\ell}=Q^{r}$ and $(R: I)_{\ell} I=I$.

Finally, consider the elements $s_{1}=\left(\begin{array}{cc}1 & 0 \\ -1 & 0\end{array}\right)$ and $s_{2}=\left(\begin{array}{ll}1 & 0 \\ 1 & 0\end{array}\right)$ of $I$ and $q_{1}=\left(\begin{array}{cc}\frac{1}{2} & -\frac{1}{2} \\ c & 0\end{array}\right)$ and $q_{2}=\left(\begin{array}{cc}\frac{1}{2} & \frac{1}{2} \\ c & 0\end{array}\right)$ of $Q$. It is easy to see that $s_{1} q_{1}, s_{2} q_{2} \notin R$ although $s_{1} q_{1}+s_{2} q_{2}=1$. On the other hand, setting $r_{1}=p_{1}=e_{1} \in I, \quad r_{2}=\left(\begin{array}{ll}0 & 0 \\ 1 & 0\end{array}\right) \in I$, and $p_{2}=\left(\begin{array}{cc}0 & 1 \\ 0 & 0\end{array}\right)$ yields $r_{1} p_{1}, r_{2} p_{2} \in R$ and $r_{1} p_{1}+r_{2} p_{2}=1$. 
In view of the previous example, we define a submodule $U$ of $Q_{R}^{r}$ to be strongly invertible if there is a submodule $M$ of ${ }_{R} Q^{r}$ such that $M U=U M=R$.

LEMMA 4.5. Let $R$ be a right and left non-singular, right and left Utumi-ring with maximal right and left ring of quotients $Q$. A submodule $U$ of $Q^{r}$ is strongly invertible if and only if it satisfies the following conditions:

i) $U$ is also a submodule of ${ }_{R} Q$.

ii) $U_{R}$ is a finitely generated projective generator of $\mathcal{M}_{R}$.

iii) ${ }_{R} U$ is a finitely generated projective generator of ${ }_{R} \mathcal{M}$.

Proof. Suppose that $U$ is strongly invertible, and choose a submodule $X$ of ${ }_{R} Q^{r}$ with $X U=U X=R$. Then, $X \subseteq(R: U)_{\ell} \cap(R: U)_{r}$ and $(R: U)_{\ell} U=R=U(R: U)_{r}$. Moreover, $R U=(U X) U=U(X U)=$ $=U R=U$ yields that $U$ is a submodule of ${ }_{R} Q^{r}$ too. By symmetry, $X$ is also a submodule of $Q_{R}^{r}$. Because of this, $(R: U)_{\ell}$ and $(R: U)_{r}$. are submodules of both $Q_{R}^{r}$ and ${ }_{R} Q^{r}$ too. Therefore, $U(R: U)_{\ell}=$ $=U(R: U)_{\ell} R=U(R: U)_{\ell} U(R: U)_{r}=U(R: U)_{r}=R$. By symmetry, $(R: U)_{r} U=R$.

By what has been shown in the last paragraph, we can write $1=u_{1} q_{1}+\ldots+u_{n} q_{n}$ with $u_{1}, \ldots, u_{n} \in U$ and $q_{1}, \ldots, q_{n} \in(R: U)_{\ell}$. Let $\phi_{i}: U \rightarrow R$ be left multiplication by $q_{i}$. As in [11, Proposition IX.5.2], the set $\left\{\left(u_{1}, \phi_{1}\right), \ldots,\left(u_{n}, \phi_{n}\right)\right\}$ is a projective basis for $U$. Select $v_{1}, \ldots, v_{m} \in U$ and $p_{1}, \ldots, p_{m} \in(R: U)_{\ell}$ with $1=p_{1} v_{1}+\ldots+p_{m} v_{m}$. Define $\psi: U^{m} \rightarrow R$ by $\psi\left(x_{1}, \ldots, x_{m}\right)=\sum_{i=1}^{m} p_{i} x_{i}$. Then, $\psi$ is onto, and $U^{m}=R \oplus W$, i.e. $U$ is a generator of $\mathcal{M}_{R}$. By symmetry, ${ }_{R} U$ is a finitely generated projective generator of $R_{R} \mathcal{M}$.

Conversely, assume that $U$ satisfies the three conditions. Observe that $(R: U)_{\ell}$ and $(R: U)_{r}$ are submodules of both $Q_{R}^{r}$ and ${ }_{R} Q^{r}$. Since it is a projective generator of $\mathcal{M}_{R}$, there is $\ell<\omega$ such that $U^{\ell}=R \oplus W$. Let $\pi: U^{\ell} \rightarrow R$ be a projection with kernel $W$, and $\delta_{j}: U \rightarrow U^{\ell}$ be the embedding into the $j^{\text {th }}$-coordinate. The map $\pi \delta_{j}: U \rightarrow R$ is left multiplication by some $q_{j} \in Q^{r}$ since $Q^{r}$ is a right self-injective ring. Clearly, since $\pi$ is onto, there are $u_{1}, \ldots, u_{\ell} \in U$ with $q_{1} u_{1}+\ldots+q_{\ell} u_{\ell}=1$. Since $q_{1}, \ldots, q_{\ell} \in(R: U)_{\ell}$, we have $(R: U)_{\ell} U=R$. By symmetry, $U(U: R)_{r}=R$. Now, $U(R: U)_{\ell}=U(R: U)_{\ell} U(R: U)_{r}=R \quad$ yields $(R: U)_{\ell} \subseteq(R: U)_{r}$. In the same way, $(R: U)_{\ell}=(R: U)_{r}$, and $U$ is strongly invertible. 
Proposition 4.6. Let $R$ be a right and left non-singular, right and left Utumi-ring. If I is a two-sided ideal of $R$ such that ${ }_{R} I$ and $I_{R}$ are finitely generated projective generators of ${ }_{R} \mathcal{M}$ and $\mathcal{M}_{R}$ respectively, then $R / I$ is projective with respect to all $R D$-exact sequences.

Proof. The proof of [7, Lemmas I.7.2 and I.7.4] can be adapted to show that $R / I$ is projective with respect to all RD-exact sequences of $R$-modules provided there are $r_{1}, \ldots, r_{n} \in R$ and $q_{1}, \ldots, q_{n} \in(R: I)_{\ell}$ such that $r_{1} q_{1}+\ldots+r_{n} q_{n}=1$ and $r_{1} q_{1}, \ldots, r_{n} q_{n} \in R$. However, this is guaranteed by Lemma 4.5.

Finally, the lattice of finitely generated right ideals over right strongly nonsingular, hereditary right and left Noetherian rings may not be distributive:

EXAMPLE 4.7. There exists a right strongly non-singular, hereditary, right and left Noetherian ring $R$ for which the lattice of right ideals is not distributive.

Proof. Let $R$ be the ring considered in Example 4.4, whose notation will be used in the following. Consider the right ideals $J=e_{1} R=\left(\begin{array}{cc}Q & 0 \\ 0 & 0\end{array}\right)$ and $K=e_{2} R=\left(\begin{array}{cc}0 & 0 \\ Q & Q\end{array}\right)$. If $I=\left(\begin{array}{ll}1 & 0 \\ 1 & 0\end{array}\right)\left(\begin{array}{ll}Q & 0 \\ Q & Q\end{array}\right)=\left\{\left(\begin{array}{cc}a & 0 \\ a & 0\end{array}\right) \mid a \in F\right\}$, then $I \cap J=I \cap K=0$, while $I \cap(J+K)=I$.

\section{REFERENCES}

[1] F. Albrecht, On projective modules over a semi-hereditary ring, Proc. Amer. Math. Soc., 12 (1961), pp. 638-639.

[2] U. AlBRECHT - A. FACCHINI, Mittag-Leffler modules over non-singular rings, Rend. Sem. Mat. Univ. Padova, 95 (1996), pp. 175-188.

[3] U. Albrecht - J. DAuns - L. Fuchs, Torsion-freeness and non-singularity over right p.p.-rings; Journal of Algebra, 285 (2005), pp. 98-119.

[4] F. Anderson - K. Fuller, Rings and Categories of Modules, Graduate Texts in Mathematics 13; Springer Verlag (1992).

[5] A.W. Chatters - C.R. Hajarnavis, Rings with Chain Conditions, Pitman Advanced Publishing 44; Boston, London, Melbourne (1980).

[6] J. DAUns - L. Fuchs, Torsion-freeness in rings with zero divisors, to appear.

[7] L. Fuchs - L. SALCE, Modules over Non-Noetherian Domains, Mathematical Surveys and Monographs 84, Amer. Math. Soc. (2000). 
[8] K. Goodearl, Ring Theory, Marcel Dekker, New York, Basel (1976).

[9] A. HatToRI, A foundation of torsion theory for modules over general rings, Nagoya Math. J., 17 (1960), pp. 147-158.

[10] J. Rotman, An Introduction to Homological Algebra; Academic Press, London (1979).

[11] B. Stenström, Rings of Quotients, Lecture Notes in Math. 217, Springer Verlag, Berlin, Heidelberg, New York (1975).

Manoscritto pervenuto in redazione il 12 gennaio 2007. 